\title{
Dynamics in a diffusive phytoplankton-zooplankton system with time delay and harvesting
}

\author{
Yanfeng Li ${ }^{1}$, Haicheng Liu', Ruizhi Yang ${ }^{2 *}$ (D) and Leiyu Tang ${ }^{3}$
}

"Correspondence:

yangruizhi529@163.com

${ }^{2}$ Department of Mathematics,

Northeast Forestry University,

Harbin, P.R. China

Full list of author information is

available at the end of the article

\section{Springer}

\begin{abstract}
The phytoplankton-zooplankton is fundamentally important to study plankton and protect marine environment. In this paper, we propose a diffusive phytoplankton-zooplankton model, in which we also consider time delay in zooplankton predation and harvesting in zooplankton. By analyzing the distribution of eigenvalues, we investigate the stability of the positive equilibrium and the existence of Hopf bifurcation using time delay as a bifurcation parameter. We analyze the property of Hopf bifurcation and give an explicit formula for determining the direction and the stability of Hopf bifurcation. Finally, by numerical simulation, we analyze the effect of harvesting, conversion rate, half-saturation, and time delay. We conclude that harvesting parameter, conversion rate, and half-saturation constant all can be used in controlling the bloom of plankton population.
\end{abstract}

MSC: 34K18; 35B32

Keywords: Phytoplankton-zooplankton; Delay; Harvesting; Stability; Hopf bifurcation

\section{Introduction}

Plankton are the basis for aquatic food chains and they can absorb not less than half of the carbon dioxide, producing a huge amount of oxygen for humans and other living animals. The importance of plankton in marine ecosystem has been widely recognized $[1,2]$. Plankton are also the base of the marine food chain. Generally, plankton can be divided into two kinds: phytoplankton and zooplankton [3]. Phytoplankton are plant species unicellular and microscopic in size. Zooplankton are animal species that feed on these phytoplankton. Therefore, zooplankton and phytoplankton form predator-prey relationship. Many researchers have studied the predator-prey model and obtained many important results [4-10].

In the last decades, the global increase in toxin-producing phytoplankton (TPP) blooms has attracted a lot of attention [11, 12]. These harmful phytoplankton may affect human health, commercial fisheries, ecosystem and environment, and so on. Toxin-producing phytoplankton can reduce zooplankton population. Some studies reveal that zooplankton's grazing and fecundity decrease in harmful algal bloom [13]. Many scholars attempt to describe the reduction of zooplankton due to toxin-producing phytoplankton [12, 14-16].

(c) The Author(s) 2019. This article is distributed under the terms of the Creative Commons Attribution 4.0 International License (http://creativecommons.org/licenses/by/4.0/), which permits unrestricted use, distribution, and reproduction in any medium, provided you give appropriate credit to the original author(s) and the source, provide a link to the Creative Commons license, and indicate if changes were made. 
Some zooplankton such as jellyfish, krill, and acetes can be harvested for food. Hence, the stocks of these tiny zooplankton play a significant role in marine reserves and fishery management. For these reasons, many scholars have studied phytoplankton-zooplankton (or predator-prey) models with harvesting [16-20]. Generally, harvesting can be divided into three types [21]: (i) constant rate of harvesting, where a fixed number of individuals are harvested per unit time; (ii) proportionate harvesting, where the catch rate is proportional to the stock and effort; and (iii) nonlinear harvesting (Holling type II, MichaelisMenten type). In [16], the authors studied a toxic phytoplankton-zooplankton model with proportionate harvesting, including the stability of equilibria and Hopf bifurcation. They suggested that harvesting can enlarge the stable range of the coexisting equilibrium to a certain extent. But when the harvesting exceeds some critical value, zooplankton will die out.

The functional response of predators to prey density is important for predator-prey models [22-25]. In ecology, many factors, such as predator hunting ability, prey escape ability, and structure of the prey habitat, can affect functional responses. Generally, functional responses can be divided into types: prey-dependent (such as Holling I-IV) and prey-predator-dependent (such as Beddington-DeAngelis, Crowley-Martin, HasselVarley). Holling IV type functional response is a kind of important functional responses and can enrich the dynamics of predator-prey models. Zhou et al. studied a predator-prey discrete-time model with Holling IV type functional response and obtained some sufficient conditions for the permanence of the system with variable coefficients [26]. Huang et al. considered a predator-prey system with Holling type IV functional response and showed that there exists a unique degenerate positive equilibrium which is a degenerate Bogdanov-Takens singularity of codimension three for other values of parameters [27]. In [28], Sharma et al. use Holling IV type functional for zooplankton to represent the effect of toxication by TPP population. They proposed a delayed phytoplankton-zooplankton model

$$
\begin{aligned}
& \dot{P}(t)=r P-a P^{2}-\frac{c P Z(t-\tau)}{P^{2} / e+P+b}, \\
& \dot{Z}(t)=\frac{s P Z}{P^{2} / e+P+b}-(d+E) Z,
\end{aligned}
$$

where $P(t)$ and $Z(t)$ represent the population density of TPP species and zooplankton at time $t$, respectively. All parameters involved with the model are positive. Parameter $r$ is the growth rate of TPP species. $c$ and $s$ are the maximum ingestion and conversion rate by zooplankton population, respectively. $a$ is the mortality rate of phytoplankton population due to intra specific competition between the species and $d$ is the natural death rate of zooplankton population. $E$ is a constant rate of harvesting. $\tau$ is time delay in zooplankton predation. The term $P /\left(P^{2} / e+P+b\right)$ represents the effect of harmful phytoplankton species on zooplankton, where $e$ is the tolerance limit of zooplankton and $b$ is the half-saturation constant. In [28], authors studied the stability and Hopf bifurcation at positive equilibrium and gave numerical simulation.

In the lakes or oceans, because of currents and turbulent diffusion or other reasons, plankton may move. Phytoplankton and zooplankton population densities are of the property of spatial variation. Many authors have modeled this spatial variation of population in phytoplankton and zooplankton by reaction diffusion equations [29-31]. Many other 
scholars have studied the predator-prey system with diffusion [32-35]. Comparing with the work without spatial variation, they suggested that diffusion may induce Turing instability and spatially non-homogeneous bifurcating periodic solution. In this paper, we will study the effect of spatial variation on model (1.1). In ocean, there are many factors that can affect the spatial variation of phytoplankton and zooplankton, but we cannot consider all these factors at the moment. We just consider the physical diffusion of the phytoplankton and zooplankton population. Assume that the water is closed with no plankton species entering and leaving at the boundary. We consider a reaction-diffusion phytoplanktonzooplankton model taking the following form:

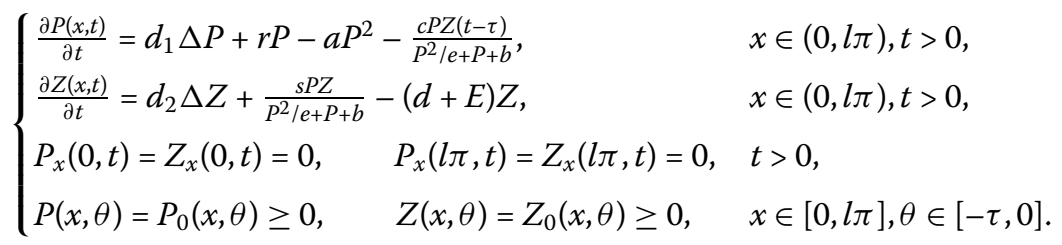

The aim of this paper is to study the dynamical properties of system (1.2). The stability of equilibrium is considered to show whether Turing instability occurs for a non-delay system and a delay system. Hopf bifurcation analysis is carried out to discuss whether the spatially homogeneous and non-homogeneous bifurcating periodic solutions exist. The effect of harvesting parameter, conversion rate, and half-saturation constant on system (1.2) are investigated to show that they can be used in controlling the bloom of plankton population.

The rest of this paper is organized as follows. In Sect. 2, we study the stability of equilibria for system (1.2) without delay. In Sect. 3, we study the effect of delay on the model including stability and Hopf bifurcation at positive equilibrium. We also study the direction and stability of the bifurcating solution by using a normal form theory and center manifold theorem. In Sect. 4, we give some numerical simulation.

\section{Stability analysis of the model without delay}

Without delay, system (1.2) becomes

$$
\begin{aligned}
& \frac{\partial P}{\partial t}=d_{1} \Delta P+r P-a P^{2}-\frac{c P Z}{P^{2} / e+P+b}, \\
& \frac{\partial Z}{\partial t}=d_{2} \Delta Z+\frac{s P Z}{P^{2} / e+P+b}-(d+E) Z .
\end{aligned}
$$

According to [28], system (1.2) has two boundary equilibria $E_{0}(0,0), E_{1}(r / a, 0)$. If the following equation

$$
P^{2}+e\left(1-\frac{s}{d+E}\right) P+e b=0
$$

has a positive root $P_{*}$ such that $P_{*}<r / a$ and $E<s-d$, then system (1.2) has a positive equilibrium $E_{*}\left(P_{*}, Z_{*}\right)$, where $Z_{*}=\frac{\left(P_{*}^{2} / e+P_{*}+b\right)\left(r-a P_{*}\right)}{c}$. In the following, we always assume that system (1.2) has a positive equilibrium $E_{*}\left(P_{*}, Z_{*}\right)$.

Define the real-valued Sobolev space

$$
X:=\left\{(u, v)^{T}: u, v \in H^{2}(0, l \pi),\left.\left(u_{x}, v_{x}\right)\right|_{x=0, l \pi}=0\right\}
$$


and the complexification of $X$ to be

$$
X_{\mathbb{C}}:=X \oplus i X=\left\{x_{1}+i x_{2} \mid x_{1}, x_{2} \in X\right\}
$$

The linearization of (1.2) near $E_{*}\left(P_{*}, Z_{*}\right)$ has the form

$$
\dot{U}(t)=d \Delta U(t)+L U(t),
$$

where $d=\operatorname{diag}\left(d_{1}, d_{2}\right)$,

$$
\begin{aligned}
& \operatorname{dom}(d \Delta)=\left\{(u, v) \in X \mid \partial_{\nu} u(t, x)=\partial_{\nu} v(t, x)=0, x=0, l \pi\right\}, \\
& L(b):=\left(\begin{array}{ll}
a_{1} & a_{2} \\
b_{1} & b_{2}
\end{array}\right),
\end{aligned}
$$

and

$$
\begin{aligned}
& a_{1}=P_{*}\left(-a+\frac{c\left(1+2 P_{*} / e\right) Z_{*}}{\left(b+P_{*}+P_{*}^{2} / e\right)^{2}}\right), \quad a_{2}=-\frac{c P_{*}}{b+P_{*}+P_{*}^{2} / e}, \\
& b_{1}=\frac{s\left(b-P_{*}^{2} / e\right)}{\left(b+P_{*}+P_{*}^{2} / e\right)^{2}}, \quad b_{2}=0 .
\end{aligned}
$$

Obviously, $a_{2}<0$. Then the characteristic equation of Eq. (2.2) is given by

$$
\lambda y-d \Delta y-L y=0 \quad \text { for some } y \in \operatorname{dom}(d \Delta) \backslash\{0\} .
$$

It is well known that the operator $u \mapsto \Delta u$ with $\partial_{\nu} u=0$ at 0 and $l \pi$ has eigenvalues $-n^{2} / l^{2}$ $\left(n \in N_{0}\right)$ with corresponding eigenfunctions $\cos (n x / l)$. Let

$$
\phi=\sum_{n=0}^{\infty}\left(\begin{array}{l}
a_{n} \\
b_{n}
\end{array}\right) \cos \left(\frac{n}{l} x\right)
$$

be an eigenfunction for $\Delta+L$ with eigenvalue $\lambda$, see also [36]. Hence, Eq. (2.4) is equivalent to the following equations:

$$
\lambda^{2}-\lambda T_{n}(b)+D_{n}(b)=0, \quad n=0,1,2, \ldots,
$$

where

$$
\left\{\begin{array}{l}
T_{n}=-\left(d_{1}+d_{2}\right) \frac{n^{2}}{l^{2}}+a_{1}, \\
D_{n}=d_{1} d_{2} \frac{n^{4}}{l^{4}}-d_{2} a_{1} \frac{n^{2}}{l^{2}}-a_{2} b_{1} .
\end{array}\right.
$$

Clearly, the roots of Eq. (2.5) are given by

$$
\lambda_{1,2}^{(n)}=\frac{1}{2}\left[T_{n} \pm \sqrt{T_{n}^{2}-4 D_{n}}\right], \quad n=0,1,2, \ldots
$$

Obviously, if

$$
\left(\mathrm{H}_{1}\right) \quad a_{1}<0 \text { and } b_{1}>0
$$


holds, then $T_{n}<0$ and $D_{n}>0$ for $n \in \mathbb{N}_{0}$, implying that the roots of Eq. (2.5) have negative real parts.

Theorem 2.1 For system (2.1), if $\left(\mathrm{H}_{1}\right)$ holds, then the equilibrium $E_{*}\left(P_{*}, Z_{*}\right)$ is locally asymptotically stable.

Remark 2.1 Similarly, we can obtain that $(0,0)$ is always unstable. If $a e r s /\left(a^{2} b e+a e r+r^{2}\right)-$ $d-E<0(>0), E_{1}(r / a, 0)$ is locally asymptotically stable (unstable). From $b_{2}=0$, we can obtain that for system (2.1), Turing instability at $E_{*}\left(P_{*}, Z_{*}\right)$ will not happen.

\section{The effect of delay on the system}

\subsection{Stability analysis and existence of Hopf bifurcation}

In the following, by analyzing the associated characteristic equation at $E_{*}\left(P_{*}, Z_{*}\right)$, we investigate the stability of $E_{*}\left(P_{*}, Z_{*}\right)$ and the existence of Hopf bifurcation for system (1.2). We assume that $\left(\mathrm{H}_{1}\right)$ always holds.

Denote

$$
\begin{aligned}
& u_{1}(t)=P(\cdot, t), \quad u_{2}(t)=Z(\cdot, t), \quad U=\left(u_{1}, u_{2}\right)^{T}, \\
& X=C\left([0, l \pi], \mathbb{R}^{2}\right), \quad \text { and } \quad \mathscr{C}_{\tau}:=C([-\tau, 0], X) .
\end{aligned}
$$

Linearizing system $(1.2)$ at $E_{*}\left(P_{*}, Z_{*}\right)$, we have

$$
\dot{U}=D \Delta U(t)+L\left(U_{t}\right)
$$

where

$$
\begin{aligned}
& D \Delta=\operatorname{diag}\left(d_{1} \Delta, d_{2} \Delta\right), \\
& \operatorname{dom}(D \Delta)=\left\{(u, v)^{T}: u, v \in C^{2}\left([0, l \pi], \mathbb{R}^{2}\right), u_{x}, v_{x}=0 \text { at } x=0, l \pi\right\},
\end{aligned}
$$

and $L: \mathscr{C}_{\tau} \mapsto X$ is defined by

$$
L\left(\phi_{t}\right)=L_{1} \phi(0)+L_{2} \phi(-\tau)
$$

for $\phi=\left(\phi_{1}, \phi_{2}\right)^{T} \in \mathscr{C}_{\tau}$ with

$$
\begin{aligned}
& L_{1}=\left(\begin{array}{ll}
a_{1} & 0 \\
b_{1} & 0
\end{array}\right), \quad L_{2}=\left(\begin{array}{cc}
0 & a_{2} \\
0 & 0
\end{array}\right), \\
& \phi(t)=\left(\phi_{1}(t), \phi_{2}(t)\right)^{T}, \quad \phi_{t}(\cdot)=\left(\phi_{1}(t+\cdot), \phi_{2}(t+\cdot)\right)^{T} .
\end{aligned}
$$

From Wu [37], we obtain that the characteristic equation for linear system (3.1) is

$$
\lambda y-d \Delta y-L\left(e^{\lambda} y\right)=0, \quad y \in \operatorname{dom}(d \Delta), y \neq 0 .
$$

It is well known that the eigenvalue problem

$$
-\varphi^{\prime \prime}=\mu \varphi, \quad x \in(0, l \pi) ; \quad \varphi^{\prime}(0)=\varphi^{\prime}(l \pi)=0
$$


has eigenvalues $\mu_{n}=n^{2} / l^{2}(n=0,1, \ldots)$ with corresponding eigenfunctions

$$
\varphi_{n}(x)=\cos \frac{n \pi}{l}, \quad n \in \mathbb{N}_{0} .
$$

Substituting

$$
y=\sum_{n=0}^{\infty}\left(\begin{array}{l}
y_{1 n} \\
y_{2 n}
\end{array}\right) \cos \frac{n \pi}{l}
$$

into the characteristic equation (3.2), it follows that

$$
\left(\begin{array}{cc}
a_{1}-d_{1} \frac{n^{2}}{l^{2}} & a_{2} e^{-\lambda \tau} \\
b_{1} & -d_{2} \frac{n^{2}}{l^{2}}
\end{array}\right)\left(\begin{array}{l}
y_{1 n} \\
y_{2 n}
\end{array}\right)=\lambda\left(\begin{array}{l}
y_{1 n} \\
y_{2 n}
\end{array}\right), \quad n=0,1, \ldots
$$

Therefore the characteristic equation (3.2) is equivalent to

$$
\Delta_{n}(\lambda, \tau)=\lambda^{2}+\lambda A_{n}+B_{n}-a_{2} b_{1} e^{-\lambda \tau}=0,
$$

where

$$
A_{n}=\left(d_{1}+d_{2}\right) \frac{n^{2}}{l^{2}}-a_{1}, \quad B_{n}=d_{1} d_{2} \frac{n^{4}}{l^{4}}-a_{1} d_{2} \frac{n^{2}}{l^{2}} .
$$

When $\tau=0$, system (1.2) becomes (2.1). If $\left(\mathrm{H}_{1}\right)$ holds, then all the roots of Eq. (3.3) with $\tau=0$ have negative real parts for $n \in \mathbb{N}_{0}$ and $\Delta_{n}(0, \tau)>0$.

We shall seek critical values of $\tau$ such that there exists a pair of simple purely imaginary eigenvalues. $i \omega(\omega>0)$ is a root of Eq. (3.3) if and only if $\omega$ satisfies

$$
-\omega^{2}+i \omega A_{n}+B_{n}-a_{2} b_{1}(\cos \omega \tau-i \sin \omega \tau)=0 .
$$

Then we have

$$
\left\{\begin{array}{l}
-\omega^{2}+B_{n}-a_{2} b_{1} \cos \omega \tau=0, \\
\omega A_{n}+a_{2} b_{1} \sin \omega \tau=0,
\end{array}\right.
$$

which leads to

$$
\omega^{4}+\omega^{2}\left(A_{n}^{2}-2 B_{n}\right)+B_{n}^{2}-a_{2}^{2} b_{1}^{2}=0
$$

Let $z=\omega^{2}$, then (3.4) can be rewritten into the following form:

$$
z^{2}+z\left(A_{n}^{2}-2 B_{n}\right)+B_{n}^{2}-a_{2}^{2} b_{1}^{2}=0
$$

and its roots are given by

$$
z_{ \pm}=\frac{1}{2}\left[-\left(A_{n}^{2}-2 B_{n}\right) \pm \sqrt{\left(A_{n}^{2}-2 B_{n}\right)^{2}-4\left(B_{n}^{2}-a_{2}^{2} b_{1}^{2}\right)}\right] .
$$


By computation, we have

$$
\begin{aligned}
& A_{n}^{2}-2 B_{n}=\left(a_{1}-d_{1} \frac{n^{2}}{l^{2}}\right)^{2}+d_{2}^{2} \frac{n^{4}}{l^{4}}>0, \\
& B_{n}-a_{2} b_{1}=d_{1} d_{2} \frac{n^{4}}{l^{4}}-a_{1} d_{2} \frac{n^{2}}{l^{2}}-a_{2} b_{1}, \\
& B_{n}+a_{2} b_{1}=d_{1} d_{2} \frac{n^{4}}{l^{4}}-a_{1} d_{2} \frac{n^{2}}{l^{2}}+a_{2} b_{1} .
\end{aligned}
$$

Obviously, $B_{n}-a_{2} b_{1}=D_{n}>0$ and $B_{0}+a_{2} b_{1}<0$ under $\left(\mathrm{H}_{1}\right)$. Since $B_{n}+a_{2} b_{1}$ is increasing with $n \in \mathbb{N}_{0}$, there exists $N_{1} \in \mathbb{N}_{0}$ such that $B_{n}+a_{2} b_{1}<0\left(n=0,1, \ldots, N_{1}\right)$ and $B_{n}+a_{2} b_{1}>0$ $\left(n>N_{1}\right)$. Hence, for $\left(n=0,1, \ldots, N_{1}\right)$, Eq. (3.5) has one positive root $z_{+}$, and Eq. (3.3) has a pair of purely imaginary roots $\pm i \omega_{n}$ at $\tau_{n}^{j}\left(j \in \mathbb{N}_{0}\right)$, where

$$
\begin{aligned}
& \omega_{n}=\sqrt{z_{+}}, \quad \tau_{n}^{j}=\tau_{n}^{0}+\frac{2 j \pi}{\omega_{n}} \quad\left(j \in \mathbb{N}_{0}\right), \\
& \tau_{n}^{0}=\tau_{n}^{0}=\frac{1}{\omega_{n}} \arccos \frac{B_{n}-\omega_{n}^{2}}{a_{2} b_{1}} .
\end{aligned}
$$

Lemma 3.1 Suppose that $\left(\mathrm{H}_{1}\right)$ is satisfied. Then $\tau_{n+1}^{j}>\tau_{n}^{j}$ for $0 \leq n \leq N_{1}$ and $j \in \mathbb{N}_{0}$ where $\tau_{n}^{j}$ is defined as in (3.6).

Proof From (3.6), we have

$$
\begin{aligned}
\omega_{n}^{2} & =\frac{1}{2}\left[\sqrt{\left(A_{n}^{2}-2 B_{n}\right)^{2}-4\left(B_{n}^{2}-a_{2}^{2} b_{1}^{2}\right)}-\left(A_{n}^{2}-2 B_{n}\right)\right] \\
& =\frac{2\left(a_{2}^{2} b_{1}^{2}-B_{n}^{2}\right)}{\sqrt{\left(A_{n}^{2}-2 B_{n}\right)^{2}-4\left(B_{n}^{2}-a_{2}^{2} b_{1}^{2}\right)}+A_{n}^{2}-2 B_{n}} \\
& =\frac{2}{\sqrt{\frac{\left(A_{n}^{2}-2 B_{n}\right)^{2}}{\left(a_{2}^{2} b_{1}^{2}-B_{n}^{2}\right)^{2}}+\frac{4}{a_{2}^{2} b_{1}^{2}-B_{n}^{2}}}+\frac{A_{n}^{2}-2 B_{n}}{a_{2}^{2} b_{1}^{2}-B_{n}^{2}}} .
\end{aligned}
$$

Under $\left(\mathrm{H}_{1}\right)$, we have $A_{n}^{2}-2 B_{n}>0$, and $a_{2}^{2} b_{1}^{2}-B_{n}^{2}>0$ for $0 \leq n \leq N_{1}$. In addition, $A_{n}^{2}-2 B_{n}$ and $B_{n}^{2}$ is strictly increasing with $n$ and $a_{2}^{2} b_{1}^{2}-B_{n}^{2}$ is strictly decreasing with $n$ for $0 \leq n \leq$ $N_{1}$. Then we have $\omega_{n+1}^{2}<\omega_{n}^{2}$ for $0 \leq n \leq N_{1}$. From (3.6), $\tau_{n+1}^{j}>\tau_{n}^{j}$ holds for $0 \leq n \leq N_{1}$.

Let $\lambda_{n}(\tau)=\alpha_{n}(\tau)+i \omega_{n}(\tau)$ be the root of (3.3) satisfying $\alpha_{n}\left(\tau_{n}^{j}\right)=0$ and $\omega_{n}\left(\tau_{n}^{j}\right)=\omega_{n}$ when $\tau$ is close to $\tau_{n}^{j}$. Then we have the following transversality condition.

Lemma 3.2 Suppose that $\left(\mathrm{H}_{1}\right)$ is satisfied. Then

$$
\alpha_{n}^{\prime}\left(\tau_{n}^{j}\right)=\left.\frac{d \lambda}{d \tau}\right|_{\tau=\tau_{n}^{j}}>0 \quad \text { for } 0 \leq n \leq N_{1} \text { and } j \in \mathbb{N}_{0}
$$

Proof Differentiating two sides of (3.3) with respect $\tau$, we have

$$
\left(\frac{d \lambda}{d \tau}\right)^{-1}=\frac{2 \lambda+A_{n}+\tau a_{2} b_{1} e^{-\lambda \tau}}{-\lambda a_{2} b_{1} e^{-\lambda \tau}} .
$$


Then

$$
\operatorname{Re}\left(\frac{d \lambda}{d \tau}\right)_{\tau=\tau_{n}^{j}}^{-1}=\frac{A_{n}^{2}-2 B_{n}+2 \omega_{n}^{2}}{a_{2}^{2} b_{1}^{2}}=\frac{\sqrt{\left(A_{n}^{2}-2 B_{n}\right)^{2}-4\left(B_{n}^{2}-a_{2}^{2} b_{1}^{2}\right)}}{a_{2}^{2} b_{1}^{2}}>0 .
$$

Therefore $\alpha_{n}^{\prime}\left(\tau_{n}^{j}\right)>0$.

Notice that $\tau_{m}^{j}=\tau_{n}^{k}$ for some $m \neq n$ may occur. In this paper, we do not consider this case. In other words, we consider

$$
\tau \in \mathcal{D}:=\left\{\tau_{n}^{j}: \tau_{m}^{j} \neq \tau_{n}^{k}, m \neq n, 0 \leq m, n \leq N_{1}, j, k \in \mathbb{N}_{0}\right\}
$$

According to the above analysis, we have the following theorem.

Theorem 3.1 For system (1.2), suppose that $\left(\mathrm{H}_{1}\right)$ holds. Then the following statements are true:

(i) If $\tau \in\left[0, \tau_{0}^{0}\right)$, then the equilibrium $P\left(u_{0}, v_{0}\right)$ is locally asymptotically stable.

(ii) If $\tau>\tau_{0}^{0}$, then the equilibrium $P\left(u_{0}, v_{0}\right)$ is unstable.

(iii) $\tau=\tau_{0}^{j}\left(j \in \mathbb{N}_{0}\right)$ are Hopf bifurcation values of system (1.2), and the bifurcating periodic solutions are spatially homogeneous, which coincide with the periodic solutions of the corresponding functional differential equation system; when $\tau \in \mathcal{D} /\left\{\tau_{0}^{k}: k \in \mathbb{N}_{0}\right\}$, system (1.2) also undergoes a Hopf bifurcation and the bifurcating periodic solutions are spatially non-homogeneous.

Remark 3.1 From Lemma 3.1, we can obtain that, for system (1.2), time delay induced Turing instability at $E_{*}\left(P_{*}, Z_{*}\right)$ will not happen.

\subsection{Stability and direction of Hopf bifurcation}

In this section, we shall study the direction of Hopf bifurcation and the stability of the bifurcating periodic solution by applying the center manifold theorem and the normal form theorem of partial functional differential equations [36, 37]. We compute the following values (see the Appendix for details of the computation):

$$
\begin{aligned}
& c_{1}(0)=\frac{i}{2 \omega_{n} \tau_{n}^{j}}\left(g_{20} g_{11}-2\left|g_{11}\right|^{2}-\frac{\left|g_{02}\right|^{2}}{3}\right)+\frac{1}{2} g_{21}, \quad \mu_{2}=-\frac{\operatorname{Re}\left(c_{1}(0)\right)}{\operatorname{Re}\left(\lambda^{\prime}\left(\tau_{n}^{j}\right)\right)}, \\
& T_{2}=-\frac{1}{\omega_{n} \tau_{n}^{j}}\left[\operatorname{Im}\left(c_{1}(0)\right)+\mu_{2} \operatorname{Im}\left(\lambda^{\prime}\left(\tau_{n}^{j}\right)\right)\right], \quad \beta_{2}=2 \operatorname{Re}\left(c_{1}(0)\right) .
\end{aligned}
$$

Theorem 3.2 For any critical value $\tau_{n}^{j}$, we have:

(i) $\mu_{2}$ determines the direction of the Hopf bifurcation: if $\mu_{2}>0$ (resp. $\left.<0\right)$, then the Hopf bifurcation is forward (resp. backward), that is, the bifurcating periodic solutions exists for $\mu>0$ (resp. $\mu<0$ ).

(ii) $\beta_{2}$ determines the stability of the bifurcating periodic solutions on the center manifold: if $\beta_{2}<0$ (resp. $>0$ ), then the bifurcating periodic solutions are orbitally asymptotically stable (resp. unstable). In particular, the stability of the bifurcating periodic solutions from the first critical value is the same as that on the center manifold. 
(iii) $T_{2}$ determines the period of bifurcating periodic solutions: if $T_{2}>0\left(\right.$ resp. $\left.T_{2}<0\right)$, then the period increases (resp. decreases).

\section{Numerical simulations}

In this section, we give some numerical simulations to suppose the theoretical findings of the present model and understand the complex dynamical behavior of the model clearly. Numerical study of this model is performed by MATLAB. Remarks (2.1) and (3.1) suggest that Turing instability at $E_{*}$ will not happen, so we just fix $d_{1}=d_{2}=1$ and $l=1$. Fix other parameters

$$
\begin{aligned}
& r=0.72, \quad a=1, \quad c=1.9, \quad e=0.25, \\
& b=1, \quad s=1.2, \quad d=0.2, \quad E=0.02 .
\end{aligned}
$$

Then system (4.2) becomes

$$
\begin{aligned}
& \frac{\partial P}{\partial t}=\Delta P+0.72 P-P^{2}-\frac{1.9 P Z}{P^{2} / 0.25+P+1}, \\
& \frac{\partial Z}{\partial t}=\Delta Z+\frac{1.2 P Z}{P^{2} / 0.25+P+1}-(0.2+0.02) Z .
\end{aligned}
$$

\subsection{Effect of harvesting}

For model (4.2), $E_{*}(0.3118,0.3654)$ is the unique positive equilibrium, and $a_{1} \approx-0.0503$, $a_{2} \approx-0.3483, b_{1} \approx 0.2536$. Then $\left(\mathrm{H}_{1}\right)$ holds and $E_{*}(0.3118,0.3654)$ is locally asymptotically stable, shown in Fig. 1 . In addition, aers $/\left(a^{2} b e+a e r+r^{2}\right)-d-E>0$ and $E_{1}(0.72,0)$ is unstable, shown in Fig. 2. From Fig. 1, we know that for model (4.2), $P(x, t)$ and $Z(x, t)$ converge to the unique positive equilibrium $E_{*}(0.3118,0.3654)$ when $t>1000$. From Fig. 2 , we know that for model (4.2), $P(x, t)$ and $Z(x, t)$ are away from the equilibrium $E_{1}(0.72,0)$ when the initial condition is near $E_{1}(0.72,0)$. These coincide with our conclusion.

If we increase harvesting rate with $E=0.03$, then $a_{1} \approx-0.0924, a_{2} \approx-0.3642, b_{1} \approx$ 0.1636 . Hence, $\left(\mathrm{H}_{1}\right)$ holds and aers $/\left(a^{2} b e+a e r+r^{2}\right)-d-E<0$. There are two positive equilibria $E_{*}(0.3601,0.3559)$ (stable), $E_{*}^{\prime}(0.6942,0.0491)$ (unstable), and a boundary equilibrium $E_{1}(0.72,0)$ (stable), shown in Fig. 3 and Fig. 4 . When $E$ crosses a certain critical value, say $E^{*}=0.04$, the positive equilibrium $E_{*}\left(P_{*}, Z_{*}\right)$ disappears, and $E_{1}(r / a, 0)$ is a unique stable equilibrium. Ecologically, it can be predicted that an increased harvesting rate of zooplankton may cause extinction of zooplankton, leaving phytoplankton alive.
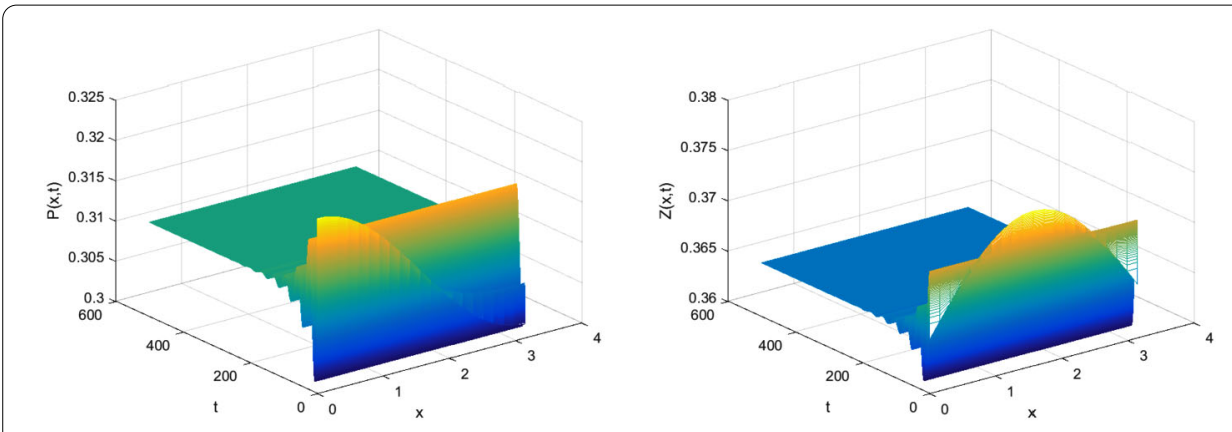

Figure 1 The numerical simulations of system (4.2) with initial condition near $E_{*}$ 


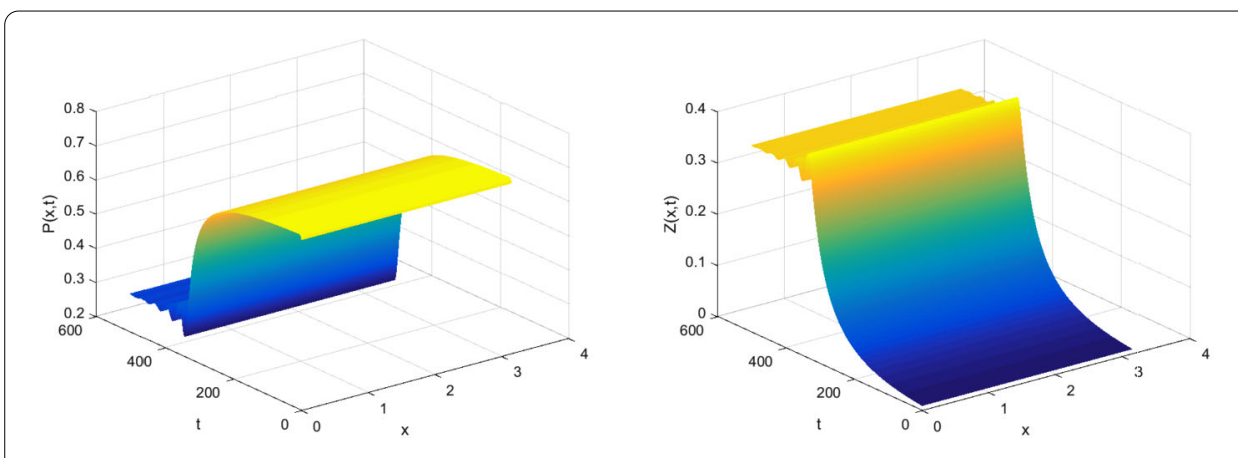

Figure 2 The numerical simulations of system (4.2) with initial condition $(0.7,0.01)$
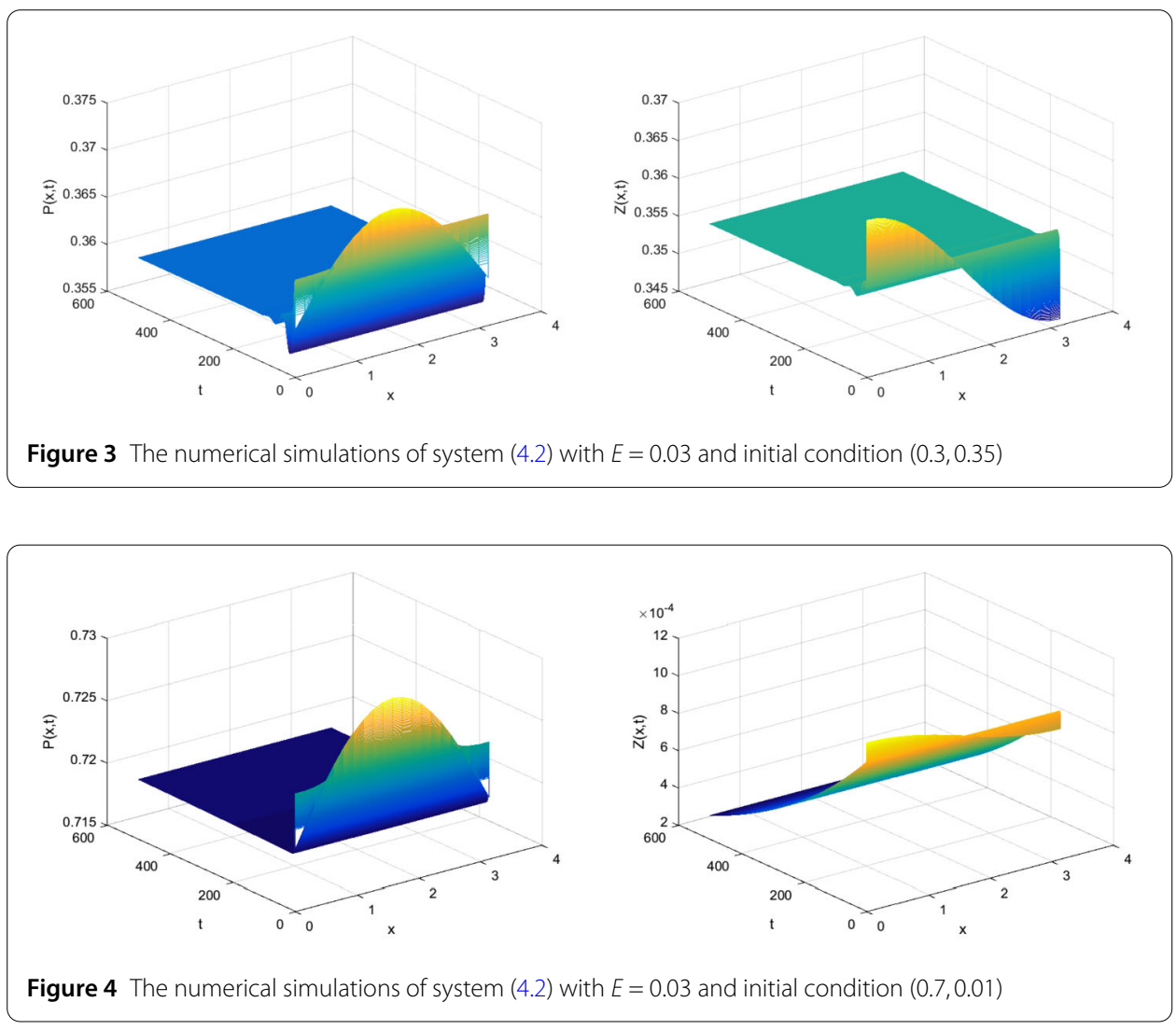

\subsection{Effect of time delay}

Under parameters (4.1), we can obtain $\tau_{0}^{0} \approx 0.5717$ and $\omega_{0} \approx 0.2951$. By Theorem 3.1(i), we know that if $\tau \in\left[0, \tau_{0}^{0}\right)$, then the equilibrium $E_{*}(0.3118,0.3654)$ is locally asymptotically stable, shown in Fig. 5. By Theorem 3.1(iii), we conclude that the equilibrium $E_{*}\left(P_{*}, Z_{*}\right)$ loses its stability and Hopf bifurcation occurs when $\tau$ crosses $\tau_{0}^{0}$. By Theorem 3.2,

$$
\mu_{2} \approx 5.9603>0, \quad \beta_{2} \approx-0.5118<0, \quad \text { and } \quad T_{2} \approx 1.1687>0 .
$$

Hence, the direction of the bifurcation is forward, the bifurcating period solutions are locally asymptotically stable, and the period of bifurcating periodic solutions increases, shown in Fig. 6. 


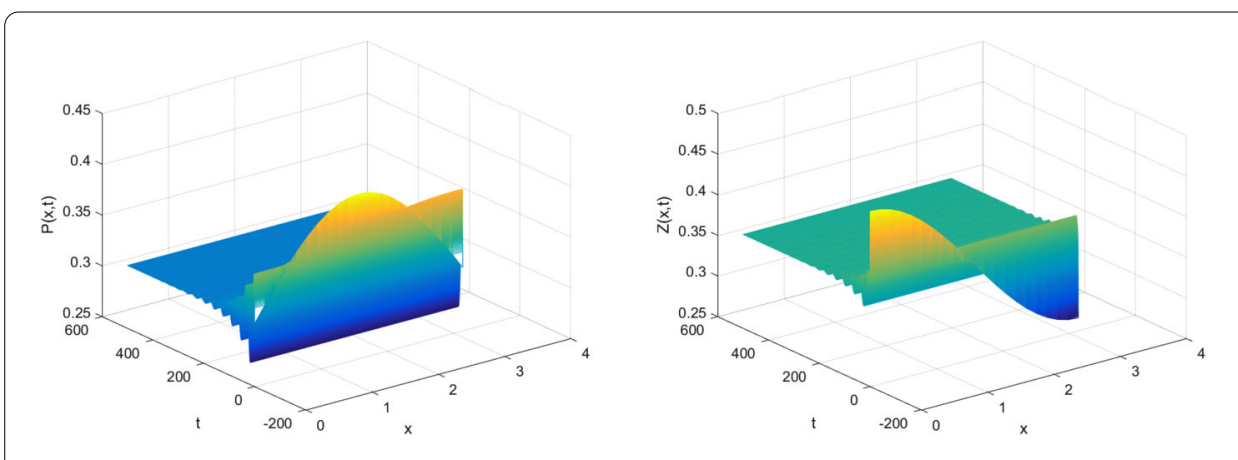

Figure 5 The numerical simulations of system (1.2) with $\tau=0.5$

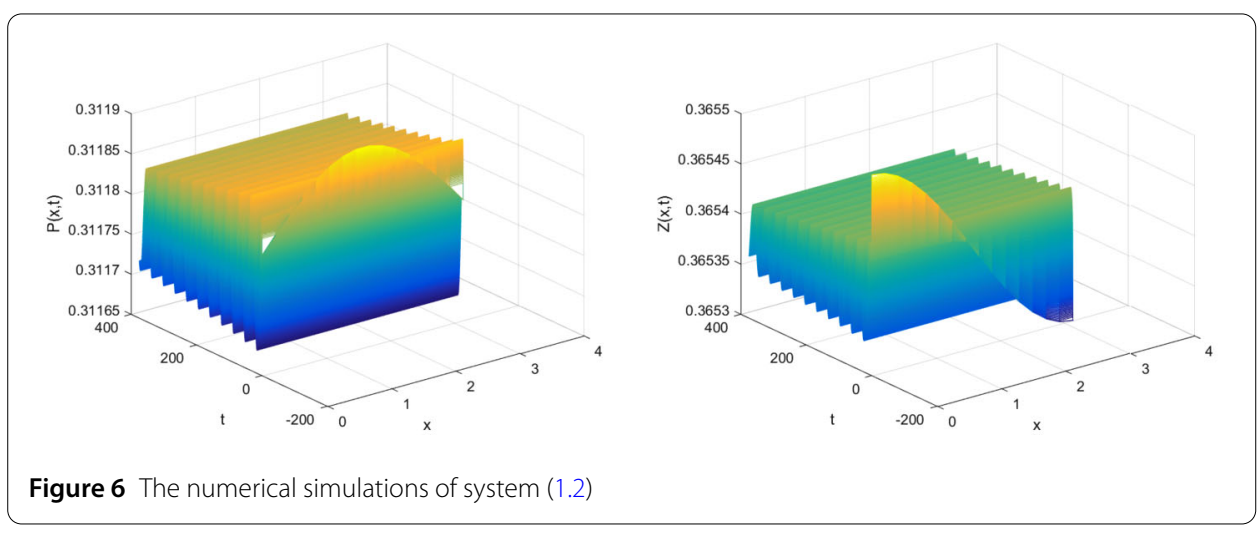

Figure 7 Bifurcation diagram for time delay and constant harvesting

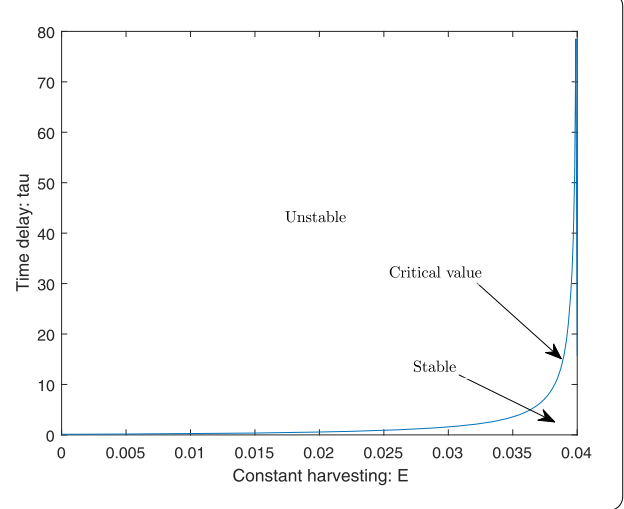

It indicates that there is a threshold limit of hunting delay below which the system does not have any excitable nature; and above it, system shows excitability in the form of oscillations.

\subsection{Bifurcation diagram analysis}

Fixing other parameters (4.1), varying the harvesting parameter and time-delay, we give a bifurcation diagram for time delay and constant harvesting, shown in Fig. 7. Appropriately increasing harvesting parameter $E$ may stabilize the unstable equilibrium $E_{*}$ (Fig. 7) of the delay system. Fixing other parameters (4.1), varying the conversion rate and time-delay, we give a bifurcation diagram for time delay and conversion rate, shown in Fig. 8. It suggests that increasing the conversion rate $s$ may destabilize the stable equilibrium $E_{*}$ of the delay 
Figure 8 Bifurcation diagram for time delay and conversion rate

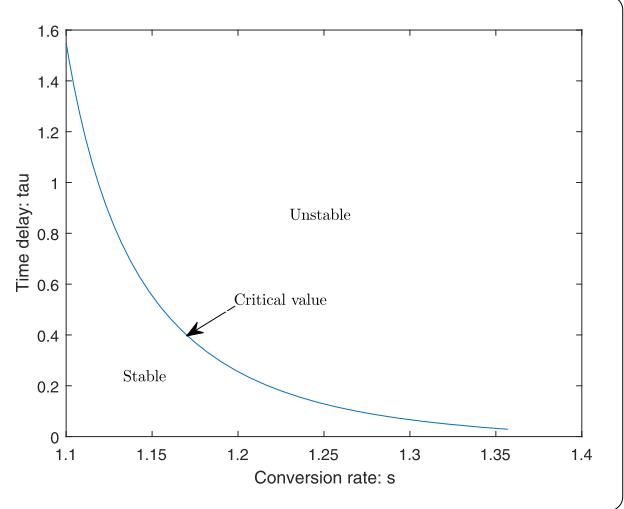

Figure 9 Bifurcation diagram for time delay and half-saturation constant

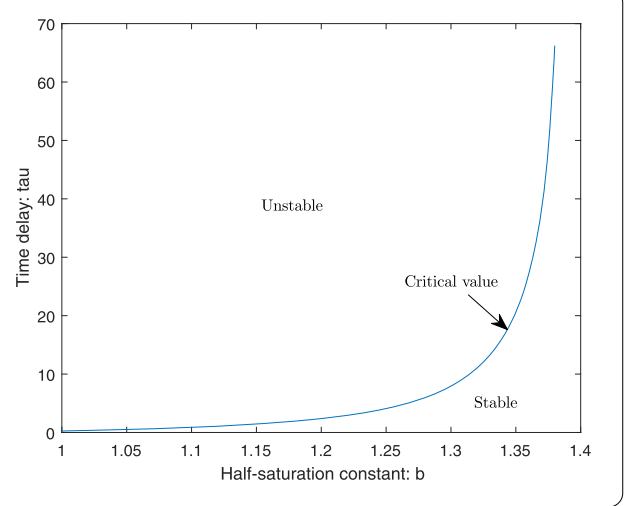

system and cause phytoplankton and zooplankton oscillation. Fixing other parameters (4.1), varying half-saturation constant and time-delay, we give a bifurcation diagram for time delay and half-saturation constant, shown in Fig. 9. It suggests that increasing halfsaturation constant $b$ may stabilize the unstable equilibrium $E_{*}$ of the delay system.

\section{Conclusion}

In this paper, we consider a diffusive phytoplankton-zooplankton model with time delay subject to the Neumann boundary condition. We study local asymptotic stability of equilibria and Hopf bifurcation at the positive equilibrium. By using the theory of normal form and center manifold, an algorithm for determining the direction and stability of Hopf bifurcation is derived.

We consider the effect of diffusion on the non-delay and delay phytoplankton-zooplankton models. For the non-delay model, we conclude that diffusion driven Turing instability cannot happen. For the delay model, we prove that $\tau_{0}^{0}$ is the minimum critical value, suggesting that Turing instability (that is, time delay induced Turing instability) cannot happen. The spatially non-homogeneous bifurcating periodic solutions induced by diffusion may occur. In addition, we studied the effect of delay on the partial differential system phytoplankton-zooplankton model. Similar to the conclusion in [28], it shows that there is a threshold limit of hunting delay below which the system does not have any excitable nature; and above it, system shows excitability in the form of oscillations. But when time delay $\tau \in \mathcal{D} /\left\{\tau_{0}^{k}: k \in \mathbb{N}_{0}\right\}$, the system undergoes a Hopf bifurcation, and the bifurcating periodic solutions are spatially non-homogeneous. 
Through numerical simulations, we show that appropriately increasing harvesting parameter $E$ may be advantageous to coexistence of phytoplankton and zooplankton. But when the harvesting parameter $E$ crosses some critical value, zooplankton may be extinct and induce phytoplankton population bloom. In [28], Sharma et al. conclude that increasing of conversion rate may cause planktonic bloom depicted through oscillation, by using numerical simulation on the non-delay ordinary differential system. For the delay partial differential system, we also get the same conclusion by analyzing bifurcation diagram for time delay and conversion rate. We also analyze bifurcation diagram for time delay and half-saturation constant, suggesting that half-saturation constant $b$ can stabilize the unstable equilibrium $E_{*}$ of the delay partial differential system. Through this study, we suggest that harvesting parameter, conversion rate, and half-saturation constant all can be used in controlling bloom of plankton population.

\section{Appendix}

Let $\tilde{P}(x, t)=P(x, \tau t)-P_{*}$ and $\tilde{Z}(x, t)=Z(x, \tau t)-Z_{*}$. For convenience, we drop the tilde. Then system (1.2) can be transformed into

$$
\left\{\begin{array}{l}
\frac{\partial u}{\partial t}=\tau\left[r\left(P+P_{*}\right)-a\left(P+P_{*}\right)^{2}-\frac{c\left(P+P_{*}\right)\left(Z(t-1)+Z_{*}\right)}{\left(P+P_{*}\right)^{2} / e+\left(P+P_{*}\right)+b}\right] \\
\frac{\partial v}{\partial t}=\tau\left[d_{2} \Delta v+\frac{s\left(P+P_{*}\right)\left(Z+Z_{*}\right)}{\left(P+P_{*}\right)^{2} / e+\left(P+P_{*}\right)+b}-(d+E)\left(Z+Z_{*}\right)\right]
\end{array}\right.
$$

for $x \in(0, l \pi)$, and $t>0$. Let

$$
\tau=\tilde{\tau}+\mu, \quad u_{1}(t)=P(\cdot, t), \quad u_{2}(t)=Z(\cdot, t), \quad \text { and } \quad U=\left(u_{1}, u_{2}\right)^{T},
$$

and $\tilde{\tau}$ is the critical value $\tau_{n}^{j}$. When $\mu=0$, system (1.2) undergoes a Hopf bifurcation at the equilibrium $(0,0)$. Then (A.1) can be rewritten in an abstract form in the phase space $\mathscr{C}_{1}:=C([-1,0], X)$

$$
\frac{d U(t)}{d t}=\tilde{\tau} D \Delta U(t)+L_{\tilde{\tau}}\left(U_{t}\right)+F\left(U_{t}, \mu\right)
$$

where $L_{\mu}(\phi)$ and $F(\phi, \mu)$ are defined by

$$
\begin{aligned}
& L_{\mu}(\phi)=\mu\left(\begin{array}{c}
a_{1} \phi_{1}(0)+a_{2} \phi_{2}(-1) \\
b_{1} \phi_{1}(0)
\end{array}\right), \\
& F(\phi, \mu)=\mu D \Delta \phi+L_{\mu}(\phi)+f(\phi, \mu),
\end{aligned}
$$

with

$$
\begin{aligned}
f(\phi, \mu)= & r\left(\phi_{1}(0)+P_{*}\right)-a\left(\phi_{1}(0)+P_{*}\right)^{2}-\frac{c\left(\phi_{1}(0)+P_{*}\right)\left(\phi_{2}(-1)+Z_{*}\right)}{\left(\phi_{1}(0)+P_{*}\right)^{2} / e+\left(\phi_{1}(0)+P_{*}\right)+b} \\
& -a_{1} \phi_{1}(0)-a_{2} \phi_{2}(-1), \\
F_{2}(\phi, \mu)= & \frac{s\left(\phi_{1}(0)+P_{*}\right)\left(\phi_{2}(0)+Z_{*}\right)}{\left(\phi_{1}(0)+P_{*}\right)^{2} / e+\left(\phi_{1}(0)+P_{*}\right)+b}-(d+E)\left(\phi_{2}(0)+Z_{*}\right)-b_{1} \phi_{1}(0),
\end{aligned}
$$

respectively, for $\phi=\left(\phi_{1}, \phi_{2}\right)^{T} \in \mathscr{C}_{1}$. 
Consider the linear equation

$$
\frac{d U(t)}{d t}=\tilde{\tau} D \Delta U(t)+L_{\tilde{\tau}}\left(U_{t}\right)
$$

According to the results in Sect. 2, we know that $\Lambda_{n}:=\left\{i \omega_{n} \tilde{\tau},-i \omega_{n} \tilde{\tau}\right\}$ are characteristic values of system (A.5) and the linear functional differential equation

$$
\frac{d z(t)}{d t}=-\tilde{\tau} D \frac{n^{2}}{l^{2}} z(t)+L_{\tilde{\tau}}\left(z_{t}\right)
$$

By Riesz representation theorem, there exists a $2 \times 2$ matrix function $\eta^{n}(\sigma, \tilde{\tau}),-1 \leq \sigma \leq 0$, whose elements are of bounded variation functions such that

$$
-\tilde{\tau} D \frac{n^{2}}{l^{2}} \phi(0)+L_{\tilde{\tau}}(\phi)=\int_{-1}^{0} d \eta^{n}(\sigma, \tau) \phi(\sigma)
$$

for $\phi \in C\left([-1,0], \mathbb{R}^{2}\right)$.

In fact, we can choose

$$
\eta^{n}(\sigma, \tau)= \begin{cases}\tau E, & \sigma=0 \\ 0, & \sigma \in(-1,0) \\ -\tau F, & \sigma=-1\end{cases}
$$

where

$$
E=\left(\begin{array}{cc}
a_{1}-d_{1} \frac{n^{2}}{l^{2}} & 0 \\
b_{1} & -d_{2} \frac{n^{2}}{l^{2}}
\end{array}\right), \quad F=\left(\begin{array}{cc}
a_{2} & 0 \\
0 & 0
\end{array}\right) .
$$

Let $A(\tilde{\tau})$ denote the infinitesimal generators of semigroup included by the solutions of Eq. (A.6) and $A^{*}$ be the formal adjoint of $A(\tilde{\tau})$ under the bilinear paring

$$
\begin{aligned}
(\psi, \phi) & =\psi(0) \phi(0)-\int_{-1}^{0} \int_{\xi=0}^{\sigma} \psi(\xi-\sigma) d \eta^{n}(\sigma, \tilde{\tau}) \phi(\xi) d \xi \\
& =\psi(0) \phi(0)+\tilde{\tau} \int_{-1}^{0} \psi(\xi+1) F \phi(\xi) d \xi
\end{aligned}
$$

for $\phi \in C\left([-1,0], \mathbb{R}^{2}\right), \psi \in C\left([-1,0], \mathbb{R}^{2}\right) . A(\tilde{\tau})$ has a pair of simple purely imaginary eigenvalues $\pm i \omega_{n} \tilde{\tau}$, and they are also eigenvalues of $A^{*}$. Let $P$ and $P^{*}$ be the center subspace, that is, the generalized eigenspace of $A(\tilde{\tau})$ and $A^{*}$ associated with $\Lambda_{n}$, respectively. Then $P^{*}$ is the adjoint space of $P$ and $\operatorname{dim} P=\operatorname{dim} P^{*}=2$.

It can be verified that $p_{1}(\theta)=(1, \xi)^{T} e^{i \omega_{n} \tilde{\tau} \sigma}(\sigma \in[-1,0]), p_{2}(\sigma)=\overline{p_{1}(\sigma)}$ is a basis of $A(\tilde{\tau})$ with $\Lambda_{n}$ and $q_{1}(r)=(1, \eta) e^{-i \omega_{n} \tilde{\tau} r}(r \in[0,1]), q_{2}(r)=\overline{q_{1}(r)}$ is a basis of $A^{*}$ with $\Lambda_{n}$, where

$$
\xi=\frac{-i l^{4} \omega b_{1}+l^{2} n^{2} b_{1} d_{2}}{l^{4} \omega^{2}+n^{4} d_{2}^{2}}, \quad \eta=\frac{i l^{2}\left(e^{i \tau \omega} a_{2}+b_{1}\right)}{l^{2} \omega+i n^{2} d_{2}} .
$$

Let $\Phi=\left(\Phi_{1}, \Phi_{2}\right)$ and $\Psi^{*}=\left(\Psi_{1}^{*}, \Psi_{2}^{*}\right)^{T}$ with

$$
\Phi_{1}(\sigma)=\frac{p_{1}(\sigma)+p_{2}(\sigma)}{2}=\left(\begin{array}{c}
\operatorname{Re}\left(e^{i \omega_{n} \tilde{\tau} \sigma}\right) \\
\operatorname{Re}\left(\xi e^{i \omega_{n} \tilde{\tau} \sigma}\right)
\end{array}\right),
$$




$$
\Phi_{2}(\sigma)=\frac{p_{1}(\sigma)-p_{2}(\sigma)}{2 i}=\left(\begin{array}{c}
\operatorname{Im}\left(e^{i \omega_{n} \tilde{\tau} \sigma}\right) \\
\operatorname{Im}\left(\xi e^{i \omega_{n} \tilde{\tau} \sigma}\right)
\end{array}\right)
$$

for $\theta \in[-1,0]$, and

$$
\begin{aligned}
& \Psi_{1}^{*}(r)=\frac{q_{1}(r)+q_{2}(r)}{2}=\left(\begin{array}{c}
\operatorname{Re}\left(e^{-i \omega_{n} \tilde{\tau} r}\right) \\
\operatorname{Re}\left(\eta e^{-i \omega_{n} \tilde{\tau} r}\right)
\end{array}\right), \\
& \Psi_{2}^{*}(r)=\frac{q_{1}(r)-q_{2}(r)}{2 i}=\left(\begin{array}{c}
\operatorname{Im}\left(e^{-i \omega_{n} \tilde{\tau} r}\right) \\
\operatorname{Im}\left(\eta e^{-i \omega_{n} \tilde{\tau} r}\right)
\end{array}\right)
\end{aligned}
$$

for $r \in[0,1]$. Then we can compute by (A.9)

$$
D_{1}^{*}:=\left(\Psi_{1}^{*}, \Phi_{1}\right), \quad D_{2}^{*}:=\left(\Psi_{1}^{*}, \Phi_{2}\right), \quad D_{3}^{*}:=\left(\Psi_{2}^{*}, \Phi_{1}\right), \quad D_{4}^{*}:=\left(\Psi_{2}^{*}, \Phi_{2}\right)
$$

Define $\left(\Psi^{*}, \Phi\right)=\left(\Psi_{j}^{*}, \Phi_{k}\right)=\left(\begin{array}{c}D_{1}^{*} D_{2}^{*} \\ D_{3}^{*} D_{4}^{*}\end{array}\right)$ and construct a new basis $\Psi$ for $P^{*}$ by

$$
\Psi=\left(\Psi_{1}, \Psi_{2}\right)^{T}=\left(\Psi^{*}, \Phi\right)^{-1} \Psi^{*}
$$

Then $(\Psi, \Phi)=I_{2}$. In addition, define $f_{n}:=\left(\beta_{n}^{1}, \beta_{n}^{2}\right)$, where

$$
\beta_{n}^{1}=\left(\begin{array}{c}
\cos \frac{n}{l} x \\
0
\end{array}\right), \quad \beta_{n}^{2}=\left(\begin{array}{c}
0 \\
\cos \frac{n}{l} x
\end{array}\right) .
$$

We also define

$$
c \cdot f_{n}=c_{1} \beta_{n}^{1}+c_{2} \beta_{n}^{2}, \quad \text { for } c=\left(c_{1}, c_{2}\right)^{T} \in \mathscr{C}_{1} \text {. }
$$

Thus the center subspace of linear equation (A.5) is given by $P_{C N} \mathscr{C}_{1} \oplus P_{S} \mathscr{C}_{1}$ and $P_{S} \mathscr{C}_{1}$ denotes the complement subspace of $P_{C N} \mathscr{C}_{1}$ in $\mathscr{C}_{1}$,

$$
\langle u, v\rangle:=\frac{1}{l \pi} \int_{0}^{l \pi} u_{1} \overline{v_{1}} d x+\frac{1}{l \pi} \int_{0}^{l \pi} u_{2} \overline{v_{2}} d x
$$

for $u=\left(u_{1}, u_{2}\right), v=\left(v_{1}, v_{2}\right), u, v \in X$ and $\left\langle\phi, f_{0}\right\rangle=\left(\left\langle\phi, f_{0}^{1}\right\rangle,\left\langle\phi, f_{0}^{2}\right\rangle\right)^{T}$.

Let $A_{\tilde{\tau}}$ denote the infinitesimal generator of an analytic semigroup induced by the linear system (A.5), and Eq. (A.1) can be rewritten as the following abstract form:

$$
\frac{d U(t)}{d t}=A_{\tilde{\tau}} U_{t}+R\left(U_{t}, \mu\right)
$$

where

$$
R\left(U_{t}, \mu\right)= \begin{cases}0, & \theta \in[-1,0) \\ F\left(U_{t}, \mu\right), & \theta=0 .\end{cases}
$$

By the decomposition of $\mathscr{C}_{1}$, the solution above can be written as

$$
U_{t}=\Phi\left(\begin{array}{l}
x_{1} \\
x_{2}
\end{array}\right) f_{n}+h\left(x_{1}, x_{2}, \mu\right)
$$


where

$$
\left(\begin{array}{l}
x_{1} \\
x_{2}
\end{array}\right)=\left(\Psi,\left\langle U_{t}, f_{n}\right\rangle\right)
$$

and

$$
h\left(x_{1}, x_{2}, \mu\right) \in P_{S} \mathscr{C}_{1}, \quad h(0,0,0)=0, \quad D h(0,0,0)=0 .
$$

In particular, the solution of (A.2) on the center manifold is given by

$$
U_{t}=\Phi\left(\begin{array}{l}
x_{1}(t) \\
x_{2}(t)
\end{array}\right) f_{n}+h\left(x_{1}, x_{2}, 0\right)
$$

Let $z=x_{1}-i x_{2}$, and notice that $p_{1}=\Phi_{1}+i \Phi_{2}$. Then we have

$$
\Phi\left(\begin{array}{l}
x_{1} \\
x_{2}
\end{array}\right) f_{n}=\left(\Phi_{1}, \Phi_{2}\right)\left(\begin{array}{c}
\frac{z+\bar{z}}{2} \\
\frac{i(z-\bar{z})}{2}
\end{array}\right) f_{n}=\frac{1}{2}\left(p_{1} z+\overline{p_{1} z}\right) f_{n}
$$

and

$$
h\left(x_{1}, x_{2}, 0\right)=h\left(\frac{z+\bar{z}}{2}, \frac{i(z-\bar{z})}{2}, 0\right) .
$$

Hence, Eq. (A.13) can be transformed into

$$
\begin{aligned}
U_{t} & =\frac{1}{2}\left(p_{1} z+\overline{p_{1} z}\right) f_{n}+h\left(\frac{z+\bar{z}}{2}, \frac{i(z-\bar{z})}{2}, 0\right) \\
& =\frac{1}{2}\left(p_{1} z+\overline{p_{1} z}\right) f_{n}+W(z, \bar{z}),
\end{aligned}
$$

where

$$
W(z, \bar{z})=h\left(\frac{z+\bar{z}}{2}, \frac{i(z-\bar{z})}{2}, 0\right) .
$$

From [37], $z$ satisfies

$$
\dot{z}=i \omega_{n} \tilde{\tau} z+g(z, \bar{z})
$$

where

$$
g(z, \bar{z})=\left(\Psi_{1}(0)-i \Psi_{2}(0)\right)\left\langle F\left(U_{t}, 0\right), f_{n}\right\rangle .
$$

Let

$$
\begin{aligned}
& W(z, \bar{z})=W_{20} \frac{z^{2}}{2}+W_{11} z \bar{z}+W_{02} \frac{\bar{z}^{2}}{2}+\cdots, \\
& g(z, \bar{z})=g_{20} \frac{z^{2}}{2}+g_{11} z \bar{z}+g_{02} \frac{\bar{z}^{2}}{2}+\cdots,
\end{aligned}
$$


from Eqs. (A.14) and (A.17), we have

$$
\begin{aligned}
u_{t}(0)= & \frac{1}{2}(z+\bar{z}) \cos \left(\frac{n x}{l}\right)+W_{20}^{(1)}(0) \frac{z^{2}}{2}+W_{11}^{(1)}(0) z \bar{z}+W_{02}^{(1)}(0) \frac{\bar{z}^{2}}{2}+\cdots \\
v_{t}(0)= & \frac{1}{2}(\xi+\bar{\xi} \bar{z}) \cos \left(\frac{n x}{l}\right)+W_{20}^{(2)}(0) \frac{z^{2}}{2}+W_{11}^{(2)}(0) z \bar{z}+W_{02}^{(2)}(0) \frac{\bar{z}^{2}}{2}+\cdots \\
v_{t}(-1)= & \frac{1}{2}\left(\xi z e^{-i \omega_{n} \tilde{\tau}}+\bar{\xi} \bar{z} e^{i \omega_{n} \tilde{\tau}}\right) \cos \left(\frac{n x}{l}\right)+W_{20}^{(2)}(-1) \frac{z^{2}}{2} \\
& +W_{11}^{(2)}(-1) z \bar{z}+W_{02}^{(2)}(-1) \frac{\bar{z}^{2}}{2}+\cdots
\end{aligned}
$$

and

$$
\begin{aligned}
\bar{F}_{1}\left(U_{t}, 0\right)= & \frac{1}{\tilde{\tau}} F_{1}=\frac{1}{2} f_{u u} u_{t}^{2}(0)+f_{u v} u_{t}(0) v_{t}(-1)+\frac{1}{2} f_{v v} v_{t}^{2}(-1) \\
& +\frac{1}{6} f_{u u u} u_{t}^{3}(0)+\frac{1}{2} f_{u u v} u_{t}^{2}(0) v_{t}(-1)+\frac{1}{2} f_{u v v} u_{t}(0) v_{t}^{2}(-1) \\
& +\frac{1}{6} f_{v v v} v_{t}^{3}(-1)+O(4), \\
\bar{F}_{2}\left(U_{t}, 0\right)= & \frac{1}{\tilde{\tau}} F_{2}=\frac{1}{2} g_{u u} u_{t}^{2}(0)+g_{u v} u_{t}(0) v_{t}(0)+\frac{1}{2} g_{v v} v_{t}^{2}(0) \\
& +\frac{1}{6} g_{u u u} u_{t}^{3}(0)+\frac{1}{2} g_{u u v} u_{t}^{2}(0) v_{t}(0)+\frac{1}{2} g_{u v v} u_{t}(0) v_{t}^{2}(0) \\
& +\frac{1}{6} g_{v v v} v_{t}^{3}(0)+O(4)
\end{aligned}
$$

with

$$
\begin{aligned}
& f_{u u}=-2 a-\frac{2 c e\left(-b e^{2}-3 b e P_{*}+P_{*}^{3}\right) Z_{*}}{\left(b e+e P_{*}+P_{*}^{2}\right)^{3}}, \quad f_{u v}=\frac{c e\left(-b e+P_{*}^{2}\right)}{\left(b e+e P_{*}+P_{*}^{2}\right)^{2}}, \\
& g_{u u}=\frac{2 e\left(-b e^{2}-3 b e P_{*}+P_{*}^{3}\right) s Z_{*}}{\left(b e+e P_{*}+P_{*}^{2}\right)^{3}}, \quad g_{u v}=-\frac{e\left(-b e+P_{*}^{2}\right) s}{\left(b e+e P_{*}+P_{*}^{2}\right)^{2}}, \\
& f_{\text {uuu }}=\frac{6 c e\left(b^{2} e^{2}-b e^{3}-4 b e^{2} P_{*}-6 b e P_{*}^{2}+P_{*}^{4}\right) Z_{*}}{\left(b e+e P_{*}+P_{*}^{2}\right)^{4}}, \\
& f_{u u v}=-\frac{c e\left(-b e^{2}-3 b e P_{*}+P_{*}^{3}\right)}{\left(b e+e P_{*}+P_{*}^{2}\right)^{3}}, \\
& g_{u u u}=-\frac{6 e\left(b^{2} e^{2}-b e^{3}-4 b e^{2} P_{*}-6 b e P_{*}^{2}+P_{*}^{4}\right) s Z_{*}}{\left(b e+e P_{*}+P_{*}^{2}\right)^{4}} \\
& g_{u u v}=\frac{e\left(-b e^{2}-3 b e P_{*}+P_{*}^{3}\right) s}{\left(b e+e P_{*}+P_{*}^{2}\right)^{3}}, \\
& f_{v v}=f_{u v v}=f_{v v v}=g_{v v}=g_{u v v}=g_{v v v}=0 .
\end{aligned}
$$

Hence,

$$
\begin{aligned}
\bar{F}_{1}\left(U_{t}, 0\right)= & \cos ^{2}\left(\frac{n x}{l}\right)\left(\frac{z^{2}}{2} \chi_{20}+z \bar{z} \chi_{11}+\frac{\bar{z}^{2}}{2} \bar{\chi}_{20}\right) \\
& +\frac{z^{2} \bar{z}}{2} \cos \left(\frac{n x}{l}\right)\left[W_{11}^{1}(0)\left(f_{u u}+e^{-i \tilde{\tau} \omega_{n}} \xi f_{u v}\right)+W_{20}^{1}(0) \frac{f_{u u}+e^{i \tilde{\tau} \omega_{n}} \bar{\xi} f_{u v}}{2}\right.
\end{aligned}
$$




$$
\begin{aligned}
& \left.+W_{11}^{2}(-1) f_{u v}+\frac{1}{2} W_{20}^{2}(-1) f_{u v}\right] \\
& +\frac{z^{2} \bar{z}}{2} \cos ^{3}\left[\frac{1}{8} f_{u u u}+\frac{1}{24}\left(e^{i \tau \omega_{n} \bar{\xi}}+2 e^{-i \tau \omega_{n}} \xi\right) f_{u u v}\right]+\cdots, \\
& \bar{F}_{2}\left(U_{t}, 0\right)=\cos ^{2}\left(\frac{n x}{l}\right)\left(\frac{z^{2}}{2} \varsigma_{20}+z \bar{z} \varsigma_{11}+\frac{\bar{z}^{2}}{2} \bar{\zeta}_{20}\right) \\
& +\frac{z^{2} \bar{z}}{2} \cos \frac{n x}{l}\left[W_{11}^{1}(0)\left(g_{u u}+\xi g_{u v}\right)\right] \\
& \left.+W_{11}^{2}(0) g_{u v}+W_{20}^{1}(0) \frac{g_{u u}+\bar{\xi} g_{u v}}{2}+\frac{1}{2} W_{20}^{2}(0) g_{u v}\right] \\
& +\frac{z^{2} \bar{z}}{2} \cos ^{3}\left[\frac{1}{8} g_{u u u}+\frac{1}{24}(\bar{\xi}+2 \xi) g_{u u v}\right]+\cdots, \\
& \left\langle F\left(U_{t}, 0\right), f_{n}\right\rangle=\tilde{\tau}\left(\bar{F}_{1}\left(U_{t}, 0\right) f_{n}^{1}+\bar{F}_{2}\left(U_{t}, 0\right) f_{n}^{2}\right) \\
& =\frac{z^{2}}{2} \tilde{\tau}\left(\begin{array}{l}
\chi_{20} \\
\varsigma_{20}
\end{array}\right) \Gamma+z \bar{z} \tilde{\tau}\left(\begin{array}{l}
\chi_{11} \\
\varsigma_{11}
\end{array}\right) \Gamma \\
& +\frac{\bar{z}^{2}}{2} \tilde{\tau}\left(\begin{array}{l}
\bar{\chi}_{20} \\
\bar{\varsigma}_{20}
\end{array}\right) \Gamma+\frac{z^{2} \bar{z}}{2} \tilde{\tau}\left(\begin{array}{l}
\kappa_{1} \\
\kappa_{2}
\end{array}\right)+\cdots
\end{aligned}
$$

with

$$
\begin{aligned}
& \Gamma=\frac{1}{l \pi} \int_{0}^{l \pi} \cos ^{3}\left(\frac{n x}{l}\right) d x, \\
& \kappa_{1}=\left[\left(f_{u u}+e^{-i \tilde{\tau} \omega_{n}} \xi f_{u v}\right) W_{11}^{(1)}(0)+f_{u v} W_{11}^{(2)}(-1)\right. \\
& \left.+\frac{1}{2}\left(f_{u u}+e^{i \tilde{\tau} \omega_{n}} \bar{\xi} f_{u v}\right) W_{20}^{(1)}(0)+\frac{1}{2}\left(f_{u v}\right) W_{20}^{(2)}(-1)\right] \frac{1}{l \pi} \int_{0}^{l \pi} \cos ^{2}\left(\frac{n x}{l}\right) d x \\
& +\left[\frac{1}{8} f_{u u u}+\frac{1}{24}\left(e^{i \tau \omega_{n}} \bar{\xi}+2 e^{-i \tau \omega_{n}} \xi\right) f_{u u v}\right] \frac{1}{l \pi} \int_{0}^{l \pi} \cos ^{4}\left(\frac{n x}{l}\right) d x, \\
& \kappa_{2}=\left[\left(g_{u u}+\xi g_{u v}\right) W_{11}^{(1)}(0)+g_{u v} W_{11}^{(2)}(0)+\frac{1}{2}\left(g_{u u}+\bar{\xi} g_{u v}\right) W_{20}^{(1)}(0)\right. \\
& \left.+\frac{1}{2} g_{u v} W_{20}^{(2)}(0)\right] \frac{1}{l \pi} \int_{0}^{l \pi} \cos ^{2}\left(\frac{n x}{l}\right) d x \\
& +\left[\frac{1}{8} g_{u u u}+\frac{1}{24}(\bar{\xi}+2 \xi) g_{u u v}\right] \frac{1}{l \pi} \int_{0}^{l \pi} \cos ^{4}\left(\frac{n x}{l}\right) d x
\end{aligned}
$$

and

$$
\begin{aligned}
& \left.\chi_{20}=\frac{1}{4} \cos ^{2}\left(\frac{n x}{l}\right)\left(f_{u u}+2 e^{-i \tilde{\tau} \omega_{n}} \xi f_{u v}\right)\right), \\
& \chi_{11}=\frac{1}{4} \cos ^{2}\left(\frac{n x}{l}\right)\left(f_{u u}+\left(e^{i \tilde{\tau} \omega_{n}} \bar{\xi}+e^{-i \tilde{\tau} \omega_{n}} \xi\right) f_{u v}\right), \\
& \varsigma_{20}=\frac{1}{4} \cos ^{2} \frac{n x}{l}\left(g_{u u}+2 \xi g_{u v}\right), \\
& \varsigma_{11}=\frac{1}{4} \cos ^{2} \frac{n x}{l}\left(g_{u u}+(\bar{\xi}+\xi) g_{u v}\right) .
\end{aligned}
$$


Denote

$$
\Psi_{1}(0)-i \Psi_{2}(0):=\left(\gamma_{1} \gamma_{2}\right)
$$

Notice that

$$
\frac{1}{l \pi} \int_{0}^{l \pi} \cos ^{3}\left(\frac{n x}{l}\right) d x=0, \quad n=1,2,3, \ldots
$$

and we have

$$
\begin{aligned}
& \left(\Psi_{1}(0)-i \Psi_{2}(0)\right)\left\langle F\left(U_{t}, 0\right), f_{n}\right\rangle \\
& =\frac{z^{2}}{2}\left(\gamma_{1} \chi_{20}+\gamma_{2} \varsigma_{20}\right) \Gamma \tilde{\tau}+z \bar{z}\left(\gamma_{1} \chi_{11}+\gamma_{2} \varsigma_{11}\right) \Gamma \tilde{\tau} \\
& \quad+\frac{\bar{z}^{2}}{2}\left(\gamma_{1} \bar{\chi}_{20}+\gamma_{2} \bar{\zeta}_{20}\right) \Gamma \tilde{\tau}+\frac{z^{2} \bar{z}}{2} \tilde{\tau}\left[\gamma_{1} \kappa_{1}+\gamma_{2} \kappa_{2}\right]+\cdots
\end{aligned}
$$

Then by (A.16), (A.18), and (A.26), we have $g_{20}=g_{11}=g_{02}=0$, for $n=1,2,3, \ldots$ If $n=0$, we have the following quantities:

$$
g_{20}=\gamma_{1} \tilde{\tau} \chi_{20}+\gamma_{2} \tilde{\tau} \varsigma_{20}, \quad g_{11}=\gamma_{1} \tilde{\tau} \chi_{11}+\gamma_{2} \tilde{\tau} \varsigma_{11}, \quad g_{02}=\gamma_{1} \tilde{\tau} \bar{\chi}_{20}+\gamma_{2} \tilde{\tau} \bar{\zeta}_{20} .
$$

And for $n \in \mathbb{N}_{0}, g_{21}=\tilde{\tau}\left(\gamma_{1} \kappa_{1}+\gamma_{2} \kappa_{2}\right)$.

Now, a complete description for $g_{21}$ depends on the algorithm for $W_{20}(0)$ and $W_{11}(0)$, which we shall compute.

From [37], we have

$$
\begin{aligned}
& \dot{W}(z, \bar{z})=W_{20} z \dot{z}+W_{11} \dot{z} \bar{z}+W_{11} z \dot{\bar{z}}+W_{02} \dot{z \bar{z}}+\cdots \\
& A_{\tilde{\tau}} W(z, \bar{z})=A_{\tilde{\tau}} W_{20} \frac{z^{2}}{2}+A_{\tilde{\tau}} W_{11} z \bar{z}+A_{\tilde{\tau}} W_{02} \frac{\bar{z}^{2}}{2}+\cdots,
\end{aligned}
$$

and $W(z, \bar{z})$ satisfies

$$
\dot{W}(z, \bar{z})=A_{\tilde{\tau}} W+H(z, \bar{z}),
$$

where

$$
\begin{aligned}
H(z, \bar{z}) & =H_{20} \frac{z^{2}}{2}+W_{11} z \bar{z}+H_{02} \frac{\bar{z}^{2}}{2}+\cdots \\
& =X_{0} F\left(U_{t}, 0\right)-\Phi\left(\Psi,\left\langle X_{0} F\left(U_{t}, 0\right), f_{n}\right\rangle \cdot f_{n}\right) .
\end{aligned}
$$

Hence, we have

$$
\left(2 i \omega_{n} \tilde{\tau}-A_{\tilde{\tau}}\right) W_{20}=H_{20}, \quad-A_{\tilde{\tau}} W_{11}=H_{11}, \quad\left(-2 i \omega_{n} \tilde{\tau}-A_{\tilde{\tau}}\right) W_{02}=H_{02},
$$

that is

$$
W_{20}=\left(2 i \omega_{n} \tilde{\tau}-A_{\tilde{\tau}}\right)^{-1} H_{20}, \quad W_{11}=-A_{\tilde{\tau}}^{-1} H_{11}, \quad W_{02}=\left(-2 i \omega_{n} \tilde{\tau}-A_{\tilde{\tau}}\right)^{-1} H_{02} .
$$


Li et al. Advances in Difference Equations

(2019) 2019:79

Page 20 of 23

(A.29)

Therefore by (A.27), for $\theta \in[-1,0)$,

$$
\begin{aligned}
& H_{20}(\theta)= \begin{cases}0, & n=1,2,3, \ldots, \\
-\frac{1}{2}\left(p_{1}(\theta) g_{20}+p_{2}(\theta) \bar{g}_{02}\right) \cdot f_{0}, & n=0,\end{cases} \\
& H_{11}(\theta)= \begin{cases}0, & n=1,2,3, \ldots, \\
-\frac{1}{2}\left(p_{1}(\theta) g_{11}+p_{2}(\theta) \bar{g}_{11}\right) \cdot f_{0}, & n=0,\end{cases} \\
& H_{02}(\theta)= \begin{cases}0, & n=1,2,3, \ldots, \\
-\frac{1}{2}\left(p_{1}(\theta) g_{02}+p_{2}(\theta) \bar{g}_{20}\right) \cdot f_{0}, & n=0,\end{cases}
\end{aligned}
$$

and

$$
H(z, \bar{z})(0)=F\left(U_{t}, 0\right)-\Phi\left(\Psi,\left\langle F\left(U_{t}, 0\right), f_{n}\right\rangle\right) \cdot f_{n},
$$

where

$$
\begin{aligned}
& H_{20}(0)= \begin{cases}\tilde{\tau}\left(\begin{array}{l}
\chi_{20} \\
\varsigma_{20}
\end{array}\right) \cos ^{2}\left(\frac{n x}{l}\right), & n=1,2,3, \ldots, \\
\tilde{\tau}\left(\begin{array}{l}
\chi_{20} \\
\varsigma_{20}
\end{array}\right)-\frac{1}{2}\left(p_{1}(0) g_{20}+p_{2}(0) \bar{g}_{02}\right) \cdot f_{0}, & n=0,\end{cases} \\
& H_{11}(0)= \begin{cases}\tilde{\tau}\left(\begin{array}{l}
\chi_{11} \\
\varsigma_{11}
\end{array}\right) \cos ^{2}\left(\frac{n x}{l}\right), & n=1,2,3, \ldots, \\
\tilde{\tau}\left(\begin{array}{l}
\chi_{11} \\
\varsigma_{11}
\end{array}\right)-\frac{1}{2}\left(p_{1}(0) g_{11}+p_{2}(0) \bar{g}_{11}\right) \cdot f_{0}, & n=0 .\end{cases}
\end{aligned}
$$

By the definition of $A_{\tilde{\tau}}$ and (A.28), we have

$$
\dot{W}_{20}=A_{\tilde{\tau}} W_{20}=2 i \omega_{n} \tilde{\tau} W_{20}+\frac{1}{2}\left(p_{1}(\theta) g_{20}+p_{2}(\theta) \bar{g}_{02}\right) \cdot f_{n}, \quad-1 \leq \theta<0 .
$$

That is,

$$
W_{20}(\theta)=\frac{i}{2 i \omega_{n} \tilde{\tau}}\left(g_{20} p_{1}(\theta)+\frac{\bar{g}_{02}}{3} p_{2}(\theta)\right) \cdot f_{n}+E_{1} e^{2 i \omega_{n} \tilde{\tau} \theta},
$$

where

$$
E_{1}= \begin{cases}W_{20}(0), & n=1,2,3, \ldots \\ W_{20}(0)-\frac{i}{2 i \omega_{n} \tilde{\tau}}\left(g_{20} p_{1}(\theta)+\frac{\bar{g}_{02}}{3} p_{2}(\theta)\right) \cdot f_{0}, & n=0 .\end{cases}
$$

Using the definition of $A_{\tilde{\tau}}$ and (A.28), we have that, for $-1 \leq \theta<0$,

$$
\begin{aligned}
& -\left(g_{20} p_{1}(0)+\frac{\bar{g}_{02}}{3} p_{2}(0)\right) \cdot f_{0}+2 i \omega_{n} \tilde{\tau} E_{1}-A_{\tilde{\tau}}\left(\frac{i}{2 \omega_{n} \tilde{\tau}}\left(g_{20} p_{1}(0)+\frac{\bar{g}_{02}}{3} p_{2}(0)\right) \cdot f_{0}\right) \\
& -A_{\tilde{\tau}} E_{1}-L_{\tilde{\tau}}\left(\frac{i}{2 \omega_{n} \tilde{\tau}}\left(g_{20} p_{1}(0)+\frac{\bar{g}_{02}}{3} p_{2}(0)\right) \cdot f_{n}+E_{1} e^{2 i \omega_{n} \tilde{\tau} \theta}\right) \\
& =\tilde{\tau}\left(\begin{array}{l}
\chi_{20} \\
\varsigma_{20}
\end{array}\right)-\frac{1}{2}\left(p_{1}(0) g_{20}+p_{2}(0) \bar{g}_{02}\right) \cdot f_{0} .
\end{aligned}
$$


As

$$
A_{\tilde{\tau}} p_{1}(0)+L_{\tilde{\tau}}\left(p_{1} \cdot f_{0}\right)=i \omega_{0} p_{1}(0) \cdot f_{0}
$$

and

$$
A_{\tilde{\tau}} p_{2}(0)+L_{\tilde{\tau}}\left(p_{2} \cdot f_{0}\right)=-i \omega_{0} p_{2}(0) \cdot f_{0}
$$

we have

$$
2 i \omega_{n} E_{1}-A_{\tilde{\tau}} E_{1}-L_{\tilde{\tau}} E_{1} e^{2 i \omega_{n}}=\tilde{\tau}\left(\begin{array}{c}
\chi_{20} \\
\varsigma_{20}
\end{array}\right) \cos ^{2}\left(\frac{n x}{l}\right), \quad n=0,1,2, \ldots
$$

That is,

$$
E_{1}=\tilde{\tau} E\left(\begin{array}{l}
\chi_{20} \\
\varsigma_{20}
\end{array}\right) \cos ^{2}\left(\frac{n x}{l}\right)
$$

where

$$
E=\left(\begin{array}{cc}
2 i \omega_{n} \tilde{\tau}+d_{1} \frac{n^{2}}{l^{2}}-a_{1} & -a_{2} e^{-2 i \omega_{n} \tilde{\tau}} \\
-b_{1} & 2 i \omega_{n} \tilde{\tau}+d_{2} \frac{n^{2}}{l^{2}}
\end{array}\right)^{-1}
$$

Similarly, from (A.29), we have

$$
-\dot{W}_{11}=\frac{i}{2 \omega_{n} \tilde{\tau}}\left(p_{1}(\theta) g_{11}+p_{2}(\theta) \bar{g}_{11}\right) \cdot f_{n}, \quad-1 \leq \theta<0 .
$$

That is,

$$
W_{11}(\theta)=\frac{i}{2 i \omega_{n} \tilde{\tau}}\left(p_{1}(\theta) \bar{g}_{11}-p_{1}(\theta) g_{11}\right)+E_{2}
$$

Similar to the procedure of computing $W_{20}$, we have

$$
E_{2}=\tilde{\tau} E^{*}\left(\begin{array}{l}
\chi_{11} \\
\varsigma_{11}
\end{array}\right) \cos ^{2}\left(\frac{n x}{l}\right)
$$

where

$$
E^{*}=\left(\begin{array}{cc}
d_{1} \frac{n^{2}}{l^{2}}-a_{1} & -a_{2} \\
-b_{1} & d_{2} \frac{n^{2}}{l^{2}}
\end{array}\right)^{-1}
$$

Thus, we can compute the following quantities which determine the direction and stability of bifurcating periodic orbits:

$$
\begin{aligned}
& c_{1}(0)=\frac{i}{2 \omega_{n} \tilde{\tau}}\left(g_{20} g_{11}-2\left|g_{11}\right|^{2}-\frac{\left|g_{02}\right|^{2}}{3}\right)+\frac{1}{2} g_{21}, \quad \mu_{2}=-\frac{\operatorname{Re}\left(c_{1}(0)\right)}{\operatorname{Re}\left(\lambda^{\prime}(\tilde{\tau})\right)}, \\
& T_{2}=-\frac{1}{\omega_{n} \tilde{\tau}}\left[\operatorname{Im}\left(c_{1}(0)\right)+\mu_{2} \operatorname{Im}\left(\lambda^{\prime}(\tilde{\tau})\right)\right], \quad \beta_{2}=2 \operatorname{Re}\left(c_{1}(0)\right) .
\end{aligned}
$$




\section{Acknowledgements}

The authors wish to express their gratitude to the editors and the reviewers for the helpful comments.

\section{Funding}

This research is supported by the National Nature Science Foundation of China (No. 11601070) and Heilongjiang Provincial Natural Science Foundation (No. A2018001).

\section{Competing interests}

The authors declare that they have no competing interests.

\section{Authors' contributions}

The idea of this research was introduced by $Y L$ and RY. All authors contributed to the main results and numerical simulations. LT contributed to revising the manuscript. All authors read and approved the final manuscript.

\section{Author details}

${ }^{1}$ Department of Mathematics, Heilongjiang Bayi Agricultural University, Daqing, P.R. China. ${ }^{2}$ Department of Mathematics, Northeast Forestry University, Harbin, P.R. China. ${ }^{3}$ College of Mathematics and Systems Science, Shandong University of Science and Technology, Qingdao, P.R. China.

\section{Publisher's Note}

Springer Nature remains neutral with regard to jurisdictional claims in published maps and institutional affiliations.

\section{Received: 27 September 2017 Accepted: 30 January 2019 Published online: 27 February 2019}

\section{References}

1. Anderson, D.M.: Turning back the harmful red tide. Nature 388(6642), 513-514 (1997)

2. Duinker, J., Wefer, G.: Das $\mathrm{CO}_{2}$-Problem und die Rolle des Ozeans. Naturwissenschaften 81 (6), 237-242 (1994)

3. Mukhopadhyay, B., Bhattacharyya, R.: Modelling phytoplankton allelopathy in a nutrient-plankton model with spatial heterogeneity. Ecol. Model. 198(1-2), 163-173 (2006)

4. Wang, J., Cheng, H., Li, Y., et al.: The geometrical analysis of a predator-prey model with multi-state dependent impulsive. J. Appl. Anal. Comput. 8(2), 427-442 (2018)

5. Jiang, Z., Wang, L.: Global Hopf bifurcation for a predator-prey system with three delays. Int. J. Bifurc. Chaos 27(7), $1750108(2017)$

6. Wang, J., Cheng, H., Meng, X.: Geometrical analysis and control optimization of a predator-prey model with multi state-dependent impulse. Adv. Differ. Equ. 2017, 252 (2017)

7. Zhang, T., Ma, W., Meng, X., Zhang, T.: Periodic solution of a prey-predator model with nonlinear state feedback control. Appl. Math. Comput. 266, 95-107 (2015)

8. Zhang, S., Meng, X., Zhang, T.: Dynamics analysis and numerical simulations of a stochastic non-autonomous predator-prey system with impulsive effects. Nonlinear Anal. Hybrid Syst. 26, 19-37 (2017)

9. Zhang, T., Meng, X., Song, Y., Zhang, T.: A stage-structured predator-prey SI model with disease in the prey and impulsive effects. Math. Model. Anal. 18(4), 505-528 (2013)

10. Meng, X., Zhao, S., Zhang, W.: Adaptive dynamics analysis of a predator-prey model with selective disturbance. Appl. Math. Comput. 266, 946-958 (2015)

11. Smayda, T.J.: Bloom dynamics: physiology, behavior, trophic effects. Limnol. Oceanogr. 42(5), 1132-1136 (1997)

12. Hallegraeff, G.: A review of harmful algae blooms and the apparent global increase. Phycologia 32(2), 79-99 (1993)

13. Kirk, K.L., Gilbert, J...: Variation in herbivore response to chemical defenses: zooplankton foraging on toxic cyanobacteria. Ecology 73(6), 2208-2217 (1992)

14. Chattopadhayay, J., Sarkar, R.R., Mandal, S.: Toxin-producing plankton may act as a biological control for planktonic blooms-field study and mathematical modelling. J. Theor. Biol. 215(3), 333-344 (2002)

15. Saha, T., Bandyopadhyay, M.: Dynamical analysis of toxin producing phytoplankton-zooplankton interactions. Nonlinear Anal., Real World Appl. 10(1), 314-332 (2009)

16. Wang, Y., Jiang, W., Wang, H.: Stability and global Hopf bifurcation in toxic phytoplankton-zooplankton model with delay and selective harvesting. Nonlinear Dyn. 73(1-2), 881-896 (2013)

17. Liu, L., Meng, X.: Optimal harvesting control and dynamics of two-species stochastic model with delays. Adv. Differ. Equ. 2017, 18 (2017)

18. Pei, Y., Li, Y., Li, C.: Evolutionary consequences of harvesting for a two-zooplankton one-phytoplankton system. Appl. Math. Model. 36(4), 1752-1765 (2012)

19. Zhang, T., Ma, W., Meng, X.: Global dynamics of a delayed chemostat model with harvest by impulsive flocculant input. Adv. Differ. Equ. 2017, 115 (2017)

20. Wang, J., Cheng, H., Liu, H., et al.: Periodic solution and control optimization of a prey-predator model with two types of harvesting. Adv. Differ. Equ. 2018, 41 (2018)

21. Upadhyay, R.K., Roy, P., Datta, J.: Complex dynamics of ecological systems under nonlinear harvesting: Hopf bifurcation and Turing instability. Nonlinear Dyn. 79(4), 2251-2270 (2014)

22. Bian, F., Zhao, W., Song, Y., Yue, R.: Dynamical analysis of a class of prey-predator model with Beddington-Deangelis functional response, stochastic perturbation, and impulsive toxicant input. Complexity 3, Article ID 3742197 (2017)

23. Feng, X., Song, Y., An, X.: Dynamic behavior analysis of a prey-predator model with ratio-dependent Monod-Haldane functional response. Open Math. 16, 623-636 (2018)

24. Liu, H., Cheng, H.: Dynamic analysis of a prey-predator model with state-dependent control strategy and square root response function. Adv. Differ. Equ. 2018, 63 (2018)

25. Feng, X., Song, Y., Liu, J., Wang, G.: Permanence, stability and coexistence of a diffusive predator-prey model with modified Leslie-Gower and B-D functional response. Adv. Differ. Equ. 2018, 314 (2018) 
26. Zhou, T., Zhang, X., Xiang, M., Wu, Z.: Permanence and almost periodic solution of a predator-prey discrete system with Holling IV functional response. Int. J. Biomath. 9, 1650035 (2016)

27. Huang, J., Xia, X., Zhang, X., Ruan, S.: Bifurcation of codimension 3 in a predator-prey system of Leslie type with simplified Holling type IV functional response. Int. J. Bifurc. Chaos 26, 1650034 (2016)

28. Sharma, A., Sharma, A.K., Agnihotri, K.: Analysis of a toxin producing phytoplankton-zooplankton interaction with Holling IV type scheme and time delay. Nonlinear Dyn. 81(1-2), 1-13 (2015)

29. Freedman, H.I., Ruan, S.: On reaction-diffusion systems of zooplankton-phytoplankton-nutrient models. Differ. Equ. Dyn. Syst. 2(7), 49-64 (1994)

30. Zhao, J., Tian, J.P., Wei, J.: Minimal model of plankton systems revisited with spatial diffusion and maturation delay. Bull. Math. Biol. 78(3), 381-412 (2016)

31. Ruan, S.: Turing instability and travelling waves in diffusive plankton models with delayed nutrient recycling. IMA J. Appl. Math. 61(1), 15-32 (1998)

32. Guin, L.N., Mandal, P.K.: Spatiotemporal dynamics of reaction-diffusion models of interacting populations. Appl. Math. Model. 38, 4417-4427 (2014)

33. Guin, L.N., Mandal, P.K.: Spatial pattern in a diffusive predator-prey model with sigmoid ratio-dependent functional response. Int. J. Biomath. 7(5), 1450047 (2014)

34. Guin, L.N., Mandal, P.K., Chakravarty, S.: Existence of spatial patterns in reaction-diffusion systems incorporating a prey refuge. Nonlinear Anal., Model. Control 20(4), 509-527 (2015)

35. Guin, L.N., Mondal, B., Chakravarty, S.: Existence of spatiotemporal patterns in the reaction-diffusion predator-prey model incorporating prey refuge. Int. J. Biomath. 9, 1-25 (2016)

36. Hassard, B.D., Kazarinoff, N.D., Wan, Y.H.: Theory and Applications of Hopf Bifurcation. Cambridge University Press, Cambridge (1981)

37. Wu, J.: Theory and Applications of Partial Functional Differential Equations. Springer, Berlin (1996)

\section{Submit your manuscript to a SpringerOpen ${ }^{\circ}$ journal and benefit from:}

- Convenient online submission

- Rigorous peer review

- Open access: articles freely available online

- High visibility within the field

- Retaining the copyright to your article

Submit your next manuscript at $\boldsymbol{\nabla}$ springeropen.com 